\title{
Is birthweight associated with total and aggressive/lethal prostate cancer risks? A systematic review and meta-analysis
}

\author{
Cindy Ke Zhou*, ${ }^{*}$, Siobhan Sutcliffe ${ }^{2}$, Judith Welsh ${ }^{3}$, Karen Mackinnon ${ }^{4}$, Diana Kuh ${ }^{4}$, Rebecca Hardy ${ }^{4}$ and \\ Michael B Cook ${ }^{1}$ \\ ${ }^{1}$ Division of Cancer Epidemiology and Genetics, National Cancer Institute, National Institutes of Health, NIH, DHHS, 9609 Medical \\ Center Drive, MSC 9774, Bethesda, MD 20892-9774, USA; ${ }^{2}$ Division of Public Health Sciences and the Siteman Cancer Center, \\ Department of Surgery, Washington University School of Medicine, St Louis, MO, USA; ${ }^{3} \mathrm{NIH}$ Library, National Institutes of Health, \\ Bethesda, MD, USA and ${ }^{4}$ Medical Research Council Unit for Lifelong Health and Ageing, University College London, London, UK
}

Background: It has been hypothesised that intrauterine exposures are important for subsequent prostate cancer risk. Prior epidemiological studies have used birthweight as a proxy of cumulative intrauterine exposures to test this hypothesis, but results have been inconsistent partly because of limited statistical power.

Methods: We investigated birthweight in relation to prostate cancer in the Medical Research Council (MRC) National Survey of Health and Development (NSHD) using Cox proportional hazards models. We then conducted a meta-analysis of birthweight in relation to total and aggressive/lethal prostate cancer risks, combining results from the NSHD analysis with 13 additional studies on this relationship identified from a systematic search in four major scientific literature databases through January 2015.

Results: Random-effects models found that per $\mathrm{kg}$ increase in birthweight was positively associated with total $(\mathrm{OR}=1.02,95 \%$ confidence interval $\left.(95 \% \mathrm{Cl})=1.00,1.05 ; \mathrm{l}^{2}=13 \%\right)$ and aggressive/lethal prostate cancer $\left(\mathrm{OR}=1.08,95 \% \mathrm{Cl}=0.99,1.19 ; \mathrm{l}^{2}=40 \%\right)$. Sensitivity analyses restricted to studies with birthweight extracted from medical records demonstrated stronger positive associations with total $\left(\mathrm{OR}=1.11,95 \% \mathrm{Cl}=1.03,1.19 ; \mathrm{P}^{2}=0 \%\right)$ and aggressive/lethal $\left(\mathrm{OR}=1.37,95 \% \mathrm{Cl}=1.09,1.74 ; \mathrm{l}^{2}=0 \%\right)$ prostate cancer. These studies heavily overlapped with those based in Nordic countries.

Conclusions: This study provides evidence that heavier birthweight may be associated with modest increased risks of total and aggressive/lethal prostate cancer, which supports the hypothesis that intrauterine exposures may be related to subsequent prostate cancer risks.

Prostate cancer is a significant public health burden and a major cause of morbidity and mortality among men worldwide. Few risk factors have been established for prostate cancer including advancing age, African ancestry, a family history of this malignancy and certain genetic polymorphisms (Al Olama et al, 2014). This may be partly owing to the fact that a majority of studies have focused on mid- to later-life exposures, and therefore may have missed putative aetiologically relevant time-windows, specifically early developmental stages of the prostate gland
(Sutcliffe and Colditz, 2013). It has been hypothesised that intrauterine exposures may contribute to subsequent increased risk of prostate cancer (Ekbom, 1998), given that the hormonal and metabolic environment during pregnancy influences the physiological development of the prostate gland and may modulate hormonal sensitivity and prostate cancer risks in adult men (Ross and Henderson, 1994; Shibata and Minn, 2000). Although the biologic mechanisms underpinning a possible in utero origin of prostate cancer are not fully understood, intrauterine sex steroid

${ }^{\star}$ Correspondence: Dr CK Zhou; E-mail: ke.zhou@nih.gov

Received 22 October 2015; revised 7 January 2016; accepted 24 January 2016; published online 1 March 2016

(c) 2016 Cancer Research UK. All rights reserved 0007-0920/16 
hormones, insulin-like growth factors (IGFs) and elevated number of fetal stem cells ('stem cell burden' hypothesis; Ekbom, 1998) have been suggested to have important roles.

Direct epidemiological evidence linking intrauterine hormones and increased stem cell numbers to prostate cancer risk is sparse, likely because of the long latency period of tumour development and the challenge of accounting for changes in environmental factors after birth. Therefore, prior epidemiological studies have used birthweight as a proxy for cumulative intrauterine exposures for multiple chronic disease outcomes (Kuh and Ben-Shlomo, 2004). For example, birthweight has been extensively studied in relation to breast cancer and a recent meta-analysis suggested a positive dose-response relationship (dos Santos Silva et al, 2008). In relation to prostate cancer - another hormonally related malignancy - an early ecologic study demonstrated that mortality rates were positively correlated with country-level birthweight (Lawson, 1998). However, results to date from epidemiological studies of birthweight and prostate cancer risks have not consistently endorsed this relationship, partly because of insufficient statistical power, especially for aggressive/lethal prostate cancers, which are of greatest clinical relevance. Therefore, we performed a cohort analysis using the Medical Research Council (MRC) National Survey of Health and Development (NSHD), which extracted birthweight from medical records within a few weeks of delivery in 1946 and has subsequently accumulated nearly 70 years of follow-up. In addition, to provide a comprehensive assessment of birthweight in relation to total and aggressive/lethal prostate cancer risks, we conducted a systematic review and metaanalysis, combining results from our smaller NSHD analysis with those prior studies identified from systematic review.

\section{MATERIALS AND METHODS}

National Survey of Health and Development. We performed a cohort analysis in the NSHD birth cohort. A detailed description of this birth cohort has been published previously (Wadsworth et al, 2006). In brief, this cohort is a socially stratified and representative sample $(N=5362)$ of single live births in England, Scotland or Wales born in March 1946 and followed up ever since. Birthweight data were extracted from medical records within a few weeks of delivery. Characteristics of cohort members and their parents have been prospectively collected at follow-ups throughout life. Cohort members have been flagged on the National Health Service Central Register since 1971 (at the age of 25 years) for notification of cancer diagnoses, deaths and emigrations. We used Cox proportional hazards models with age as the underlying time scale to estimate hazard ratio (HRs) and 95\% confidence intervals (CIs) of continuous (per $\mathrm{kg}$ ) and categorical birthweight $(<3000,3000$ $3499,3500-3999$ and $\geqslant 4000 \mathrm{~g}$ ) in relation to total prostate cancer. Follow-up of this analysis started at the age of 25 years when cancer register data became available, and continued until prostate cancer diagnosis, death, emigration or age 67 years (i.e., until November 2014, the most recent date of available cancer registry data), whichever occurred earlier. We included 2791 men in this analysis, after excluding 2547 females, 17 men without birthweight information and 7 without follow-up information.

Literature search. To complement the NSHD analysis and increase the statistical power, we also conducted a systematic review on the relationship between birthweight and prostate cancer risks. Four major scientific literature databases (PubMed (National Centre for Biotechnology Information, US National Library of Medicine, Bethesda, MD, USA), EMBASE (Elsevier BV, Amsterdam, The Netherlands), Scopus (Elsevier BV), Web of Science (Thomson Reuters, New York, NY, USA)) were systematically searched through January 2015 by a trained clinical informationist (JW).
No date or language restrictions were applied. Search terms incorporated a wide array of variables for, or related to, birthweight and prostate cancer or cancer in general. Search strategies are available from the authors on request. Two reviewers (CKZ and $\mathrm{MBC}$ ) independently assessed titles, abstracts and keywords to select potentially relevant studies from retrieved references. Discordant selections were resolved by consensus. The full text of any original contribution that indicated a potential to contain data on birthweight and prostate cancer, or of any review on prostate cancer aetiology, was retrieved. Bibliographies of retrieved full-text articles were also reviewed to identify references that may have been missed or absent from the databases used. We contacted corresponding authors for additional data if their publications did not present adequate information necessary for meta-analysis. References were compiled and managed using Endnote X7. To be included in this meta-analysis, a published study had to fulfill the following criteria: (1) study populations were born as singletons; (2) have at least 20 cases and 20 controls in the analysis; (3) used a cohort or case-control study design; and (4) provided risk estimates with $95 \%$ CIs per unit change in birthweight, or tabulated counts of cases and controls by birthweight category to allow for log-linear coefficients and standard errors to be estimated using methods described previously (Chene and Thompson, 1996). When multiple reports were published with substantial geotemporal overlap in the base population, we included the most recent or best-powered publication. Two reviewers (CKZ and $\mathrm{MBC}$ ) independently extracted data from selected articles according to a standard form created a priori for this study. Differences were resolved by consensus.

Meta-analysis. For studies reporting results with categorical birthweight, we calculated individual unadjusted log odds ratios (ORs) per $\mathrm{kg}$ increase in birthweight and their standard errors using tabulated counts of cases and controls by birthweight category via methods described previously (Chene and Thompson, 1996). For studies reporting results with continuous birthweight, we back-calculated log risk estimates per $\mathrm{kg}$ and their standard errors using reported estimates and their 95\% CIs. Unadjusted or minimally adjusted risk estimates were preferred over maximally adjusted estimates, given the fact that putative confounding factors showed little or no effect on estimates attained for the relationship between birthweight and prostate cancer risks in prior studies. This has the additional benefit of reducing excess heterogeneity attributable to different model specifications used in published studies. We pooled study-specific risk estimates using a randomeffects meta-analytic model, and tested for between-study heterogeneity in risk estimates using a $\chi^{2}$ test based on the Q statistic, as well as the $I^{2}$ statistic and its $95 \%$ uncertainty interval (95\% UI). To evaluate publication bias, we visually inspected the symmetry of funnel plots and quantitatively tested the bias using Begg's rank correlation test and Egger's linear regression test. To evaluate the influence of individual studies on the summary estimates, we performed influence analyses by leaving out one study at a time and re-estimating the summary estimates. We also performed meta-regression by separately including study-level covariates, such as birthweight source (medical records $v s$ reported), country (Nordic vs non-Nordic), design (cohort/nested case-control vs population-based case-control), mid-birth year ( $\leqslant 1945$ vs $>1945$; The World War II ends in 1945), mean/median age at diagnosis ( $\leqslant 65$ vs $>65$ years) and adjustment status (no/minimal vs maximum), which were specified a priori. Finally, analyses were also conducted using fixed-effects models for the purpose of comparison and comprehensiveness. Meta-analyses were conducted separately for total and aggressive/lethal prostate cancers. All statistical analyses were performed using Stata version 13 (Stata Corp., College Station, TX, USA). Two-sided $P$-values $<0.05$ were considered statistically significant. 


\section{RESULTS}

NSHD results. Of the 2791 men in the NSHD birth cohort, 81 were diagnosed with prostate cancer through November 2014, of which $24(30 \%)$ were diagnosed at ages younger than 60 years. Characteristics by event status are shown in Supplementary Table 1. Generally, these characteristics were evenly distributed by event status, except that fathers and maternal grandfathers of prostate cancer cases were more likely to have non-manual jobs, and mothers were more likely to have higher education compared with those of controls. Hazard ratios and 95\% CIs estimated from Cox proportional hazards models are shown in Supplementary Table 2. In this NSHD analysis, neither continuous $\left(\mathrm{OR}_{\text {per } \mathrm{kg} \text { increase }}=0.84,95 \% \mathrm{CI}=0.56,1.27\right)$ nor categorical $\left(\mathrm{OR}_{3000-3499}\right.$ vs $<3000 \mathrm{~g}=1.03,95 \% \mathrm{CI}=0.54,1.95 ; \mathrm{OR}_{3500-3999}$ s $<3000 \mathrm{~g}=0.98,95 \% \mathrm{CI}=0.51,1.86 ; \mathrm{OR}_{\geqslant} 4000$ s $<3000 \mathrm{~g}=0.93,95 \%$ $\mathrm{CI}=0.42,2.06) \quad$ birthweight were significantly associated with prostate cancer. The proportional hazards assumptions held with nonsignificant Schoenfeld residuals test $\left(P_{\text {continuous }}\right.$ birthweight $=0.103 ; \quad P_{\text {categorical }}$ birthweight $\left.=0.478\right)$. As adjustment for father's and maternal grandfather's occupation as well as mother's education did not materially change the results (Supplementary Table 2), we used unadjusted risk estimates in subsequent meta-analysis.

Literature search and study characteristics. A flow chart for this systematic review is shown in Figure 1. After independently screening titles, abstracts and key words, we deemed 49 articles to be potentially eligible and retrieved their full texts. After reviewing the full texts and their bibliographies, we included 14 studies in total (Ekbom et al, 1996, 2000; Boland et al, 2003; Kajantie et al, 2005; McCormack et al, 2005; Nilsen et al, 2005; Eriksson et al, 2007; Sutcliffe et al, 2007; Parent et al, 2008; Barker et al, 2012;
Lope et al, 2012; Cook et al, 2013; Gerdtsson et al, 2015) including the NSHD analysis presented herein for qualitative and quantitative synthesis, 8 of which were cohort in design (Kajantie et al, 2005; McCormack et al, 2005; Nilsen et al, 2005; Eriksson et al, 2007; Sutcliffe et al, 2007; Barker et al, 2012; Cook et al, 2013) including the NSHD analysis presented herein, 3 were nested casecontrol (Ekbom et al, 1996, 2000; Gerdtsson et al, 2015) and 3 were population-based case-control (Boland et al, 2003; Parent et al, 2008; Lope et al, 2012). Four of these 14 included studies used reported birthweight (Sutcliffe et al, 2007; Parent et al, 2008; Lope et al, 2012; Cook et al, 2013), 9 were conducted in Nordic countries (Ekbom et al, 1996, 2000; Kajantie et al, 2005; McCormack et al, 2005; Nilsen et al, 2005; Eriksson et al, 2007; Barker et al, 2012; Cook et al, 2013; Gerdtsson et al, 2015), and 8 reported the association between birthweight and aggressive/lethal prostate cancers (Ekbom et al, 1996; Kajantie et al, 2005; Nilsen et al, 2005; Eriksson et al, 2007; Sutcliffe et al, 2007; Parent et al, 2008; Lope et al, 2012; Gerdtsson et al, 2015). Among those eight studies with subtype results, the Finnish study by Kajantie et al (2005) was the only study that did not report the association with total incident prostate cancer. Among excluded studies, five overlapped with the base populations of included studies (Tibblin et al, 1995; Platz et al, 1998; Ahlgren et al, 2007; Lahmann et al, 2010, 2012). Characteristics of included studies on birthweight in relation to prostate cancer risks are shown in Table 1.

\section{Meta-analysis}

Total prostate cancer. We pooled risk estimates from 13 studies for the risk of total prostate cancer per $\mathrm{kg}$ increase in birthweight using a random-effects model, and found that birthweight was associated with a small increased risk of the outcome $(\mathrm{OR}=1.02$, 95\% CI $=1.00,1.05 ; P=0.045$ ) (Figure 2). Publication bias was unlikely given the nonsignificant Begg's $(P=0.583)$ and Egger's

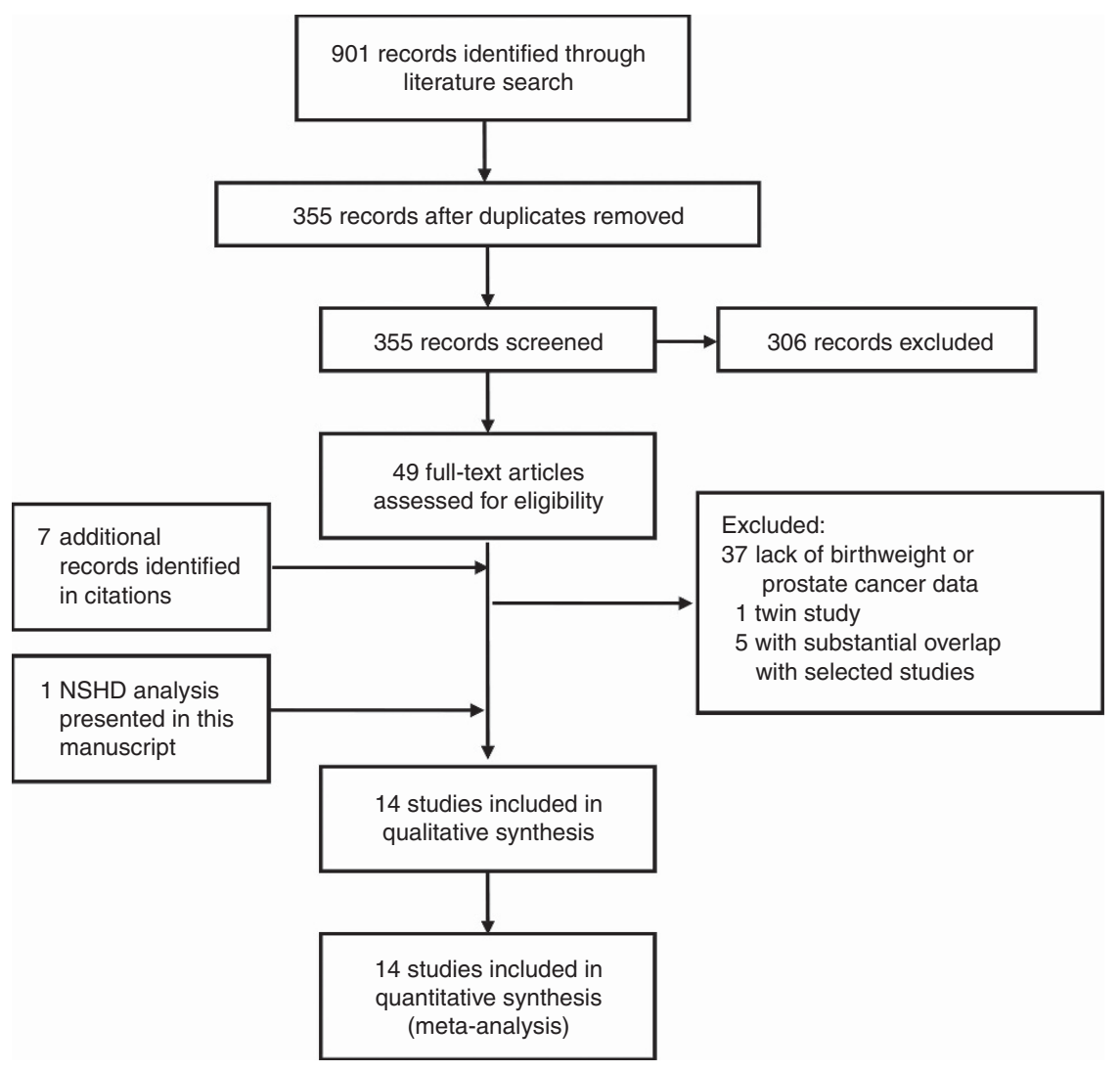

Figure 1. Flow chart for systematic review. 


\begin{tabular}{|c|c|c|c|c|c|c|c|c|c|c|c|}
\hline 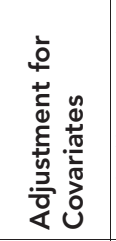 & & 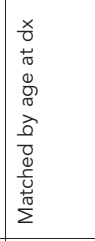 & 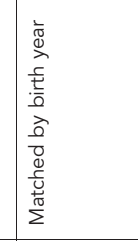 & 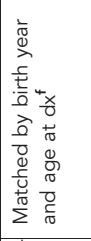 & 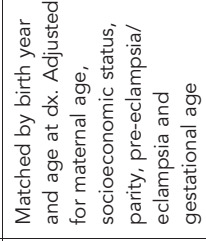 & 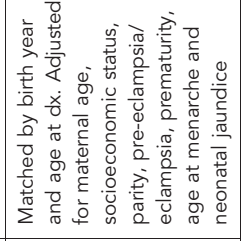 & 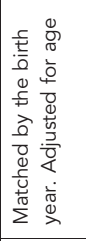 & 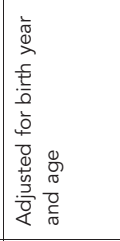 & 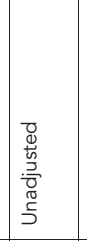 & 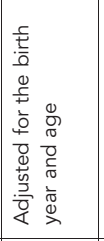 & 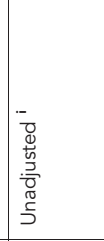 \\
\hline 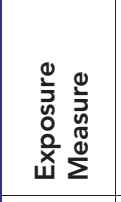 & 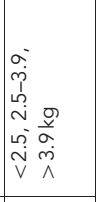 & 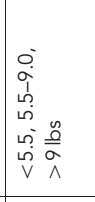 & 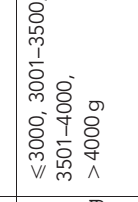 & 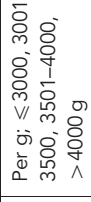 & \begin{tabular}{|l} 
व) \\
aे \\
2
\end{tabular} & $\begin{array}{l}\frac{\pi}{a} \\
\frac{a}{2}\end{array}$ & 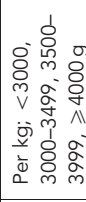 & $\begin{array}{l}2 \\
\frac{2}{2} \\
\frac{a}{2} \\
\frac{2}{2}\end{array}$ & 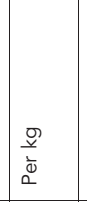 & 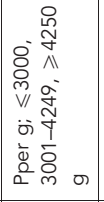 & 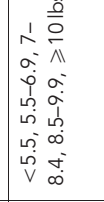 \\
\hline 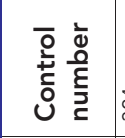 & & 哈 & 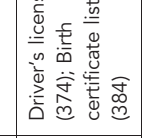 & 品 & 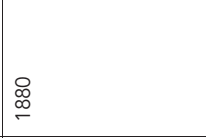 & S్ & $\mathbb{z}$ & $\frac{\pi}{2}$ & $\frac{\mathbb{s}}{2}$ & $\frac{\pi}{z}$ & $\frac{\pi}{z}$ \\
\hline $\begin{array}{l}x \\
\text { a } \\
0 \\
0 \\
0 \\
8 \\
8\end{array}$ & 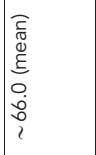 & 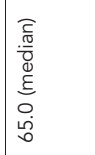 & 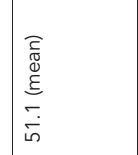 & 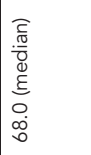 & & 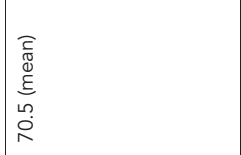 & | & 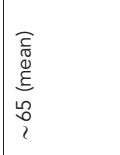 & 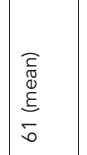 & & 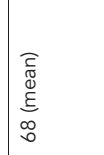 \\
\hline 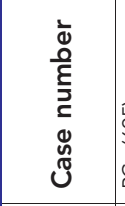 & 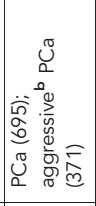 & 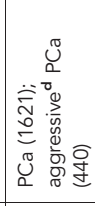 & 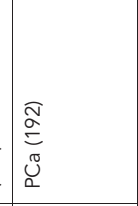 & 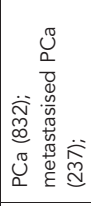 & 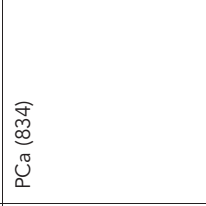 & 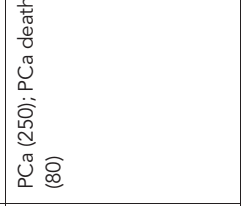 & 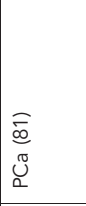 & 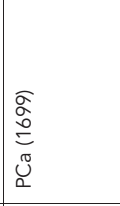 & 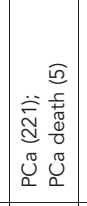 & 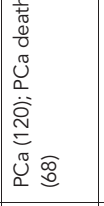 & 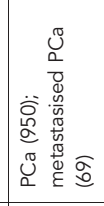 \\
\hline 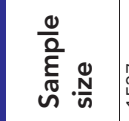 & & 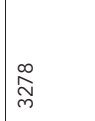 & 足 & 罗 & $\underset{\substack{n\\
}}{5}$ & 类 & 京 & 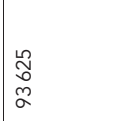 & 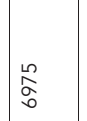 & 桑 & 莒 \\
\hline 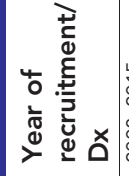 & & 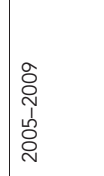 & 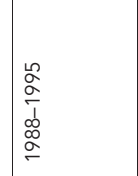 & 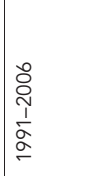 & 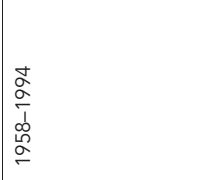 & 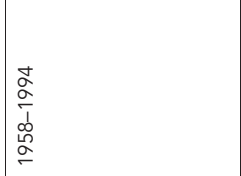 & 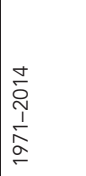 & 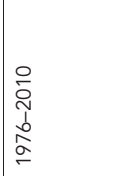 & 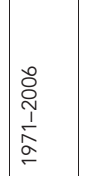 & & 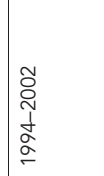 \\
\hline 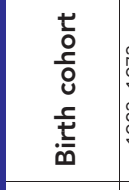 & $\begin{array}{l}\frac{1}{2} \\
\frac{2}{2} \\
\frac{2}{2} \\
\frac{2}{2}\end{array}$ & 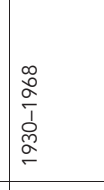 & 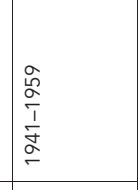 & 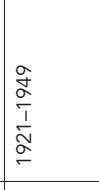 & 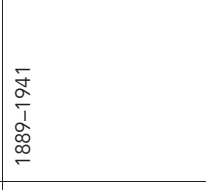 & 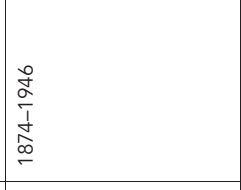 & $\frac{d}{d 0}$ & 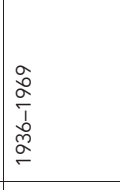 & 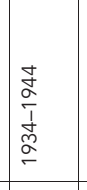 & $\stackrel{m}{d}$ & $\begin{array}{l}-1 \\
\frac{\alpha}{2} \\
\frac{1}{2} \\
\frac{1}{2} \\
\end{array}$ \\
\hline 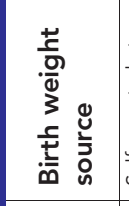 & 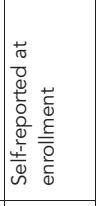 & 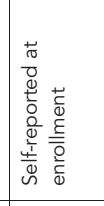 & 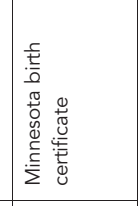 & 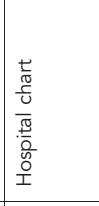 & 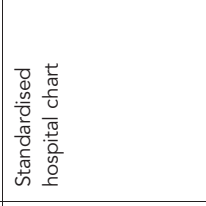 & 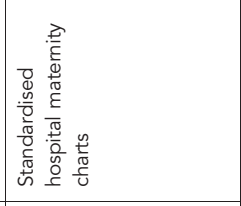 & 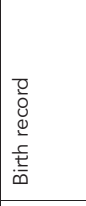 & 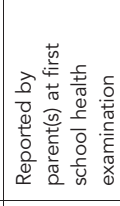 & 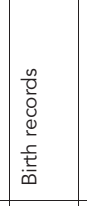 & 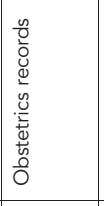 & 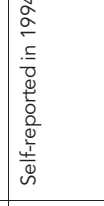 \\
\hline 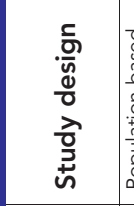 & 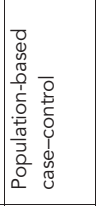 & 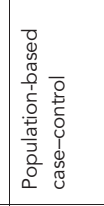 & 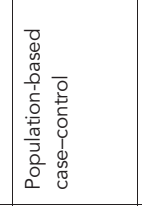 & 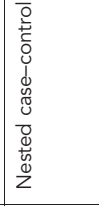 & 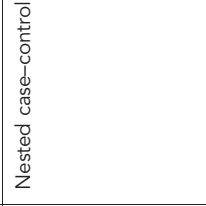 & 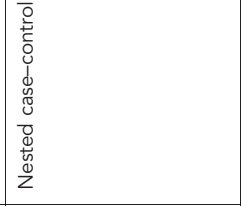 & 憘 & $\begin{array}{l}\frac{\mathrm{t}}{\frac{5}{0}} \\
\text { s. }\end{array}$ & $\begin{array}{l}\frac{t}{0} \\
\frac{0}{0} \\
\end{array}$ & 憘 & $\begin{array}{l}\frac{t}{0} \\
\frac{0}{8} \\
\end{array}$ \\
\hline 总总 & & 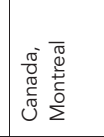 & 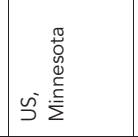 & 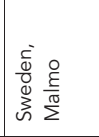 & 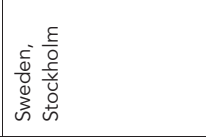 & 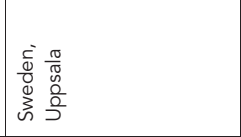 & 兰 & 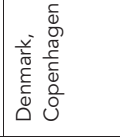 & 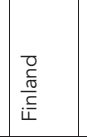 & 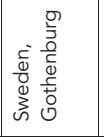 & s \\
\hline 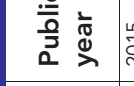 & & 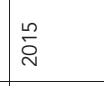 & : & 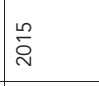 & : & 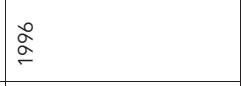 & 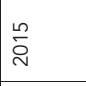 & 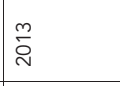 & స̃ & 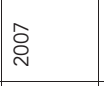 & 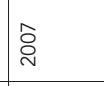 \\
\hline 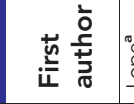 & & 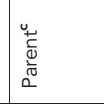 & 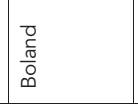 & 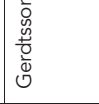 & 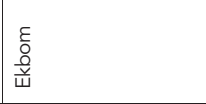 & \begin{tabular}{|l}
$\frac{5}{0}$ \\
$\frac{0}{4}$
\end{tabular} & 敦 & 总 & 离 & $\begin{array}{l}\text { 总 } \\
\text { 業 } \\
\end{array}$ & \begin{tabular}{|l}
$\frac{0}{5}$ \\
$\frac{5}{5}$ \\
5
\end{tabular} \\
\hline
\end{tabular}




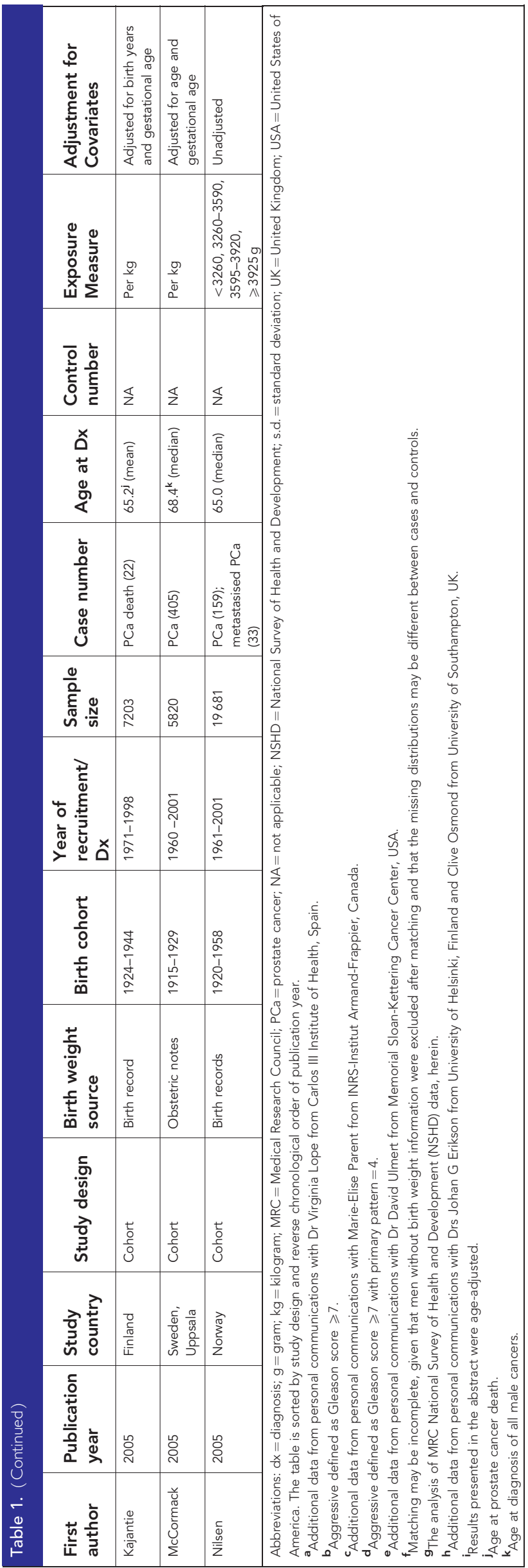

$(P=0.202)$ tests, although the funnel plot appeared slightly asymmetric (Supplementary Figure 1). Omission of one study at a time did not materially change the summary risk estimate (Supplementary Table 3). In particular, when Sutcliffe et al (2007) or Parent et al (2008) - which had the greatest weights - were excluded, the summary risk estimates did not deviate from the overall risk estimate. Subgroup analyses of study-level variables showed that birthweight source $(P=0.014)$ and country $(P=0.005)$ may partially explain the small amount of betweenstudy heterogeneity detected, with higher summary risk estimates for studies that used medical records to ascertain birthweight $\left(\mathrm{OR}_{\text {per } \mathrm{kg} \text { increase }}=1.11,95 \% \mathrm{CI}=1.03,1.19\right)$ and studies in Nordic countries $\left(\mathrm{OR}_{\text {per kg increase }}=1.10,95 \% \mathrm{CI}=1.04,1.16\right)$ (Table 2). Seven of the nine studies that used medical records to ascertain birthweight were based in Nordic countries. Given the heterogeneity test was not statistically significant $(P=0.312)$ with $I^{2}=13 \%(95 \% \mathrm{UI}=0 \%, 52 \%)$, we performed sensitivity analyses using fixed-effects models, which provided similar overall $\left(\mathrm{OR}_{\mathrm{per}} \mathrm{kg}\right.$ increase $=1.02,95 \% \mathrm{CI}=1.00,1.03 ; P=0.028)$ and subgroup summary estimates (Supplementary Table 4 ).

Aggressive/lethal prostate cancer. We separately conducted a meta-analysis using a random-effects model of eight studies that provided birthweight in relation to aggressive/lethal prostate cancer risk. We found that per $\mathrm{kg}$ increase in birthweight seemed to be associated with an $8 \%$ increased risk of aggressive/lethal disease $(\mathrm{OR}=1.08,95 \% \mathrm{CI}=0.99,1.18 ; P=0.076)$, although the association was not statistically significant (Figure 3). Publication bias was suggested by the asymmetry of the funnel plot (Supplementary Figure 2), yet objective judgment is difficult with only eight studies and Begg's $(P=0.266)$ and Egger's $(P=0.054)$ tests were not statistically significant. In influence analyses, the study by Parent et al (2008) was found to have an influence on the summary estimate, as the estimate became statistically significant and increased by $6 \%(\mathrm{OR}=1.14,95 \% \mathrm{CI}=1.01,1.28)$ once such study was removed (Supplementary Table 3). Of the eight studies included in this analysis, all Nordic studies had retrieved birthweight information from medical records, whereas all nonNordic studies used reported birthweight. Subgroup analyses of the study-level variables showed that the moderate between-study heterogeneity $\left(I^{2}=40 \%, 95 \% \mathrm{UI}=0 \%, 73 \% ; P=0.115\right)$ may be explained by birthweight source/study country $(P=0.020)$ and study design $(P=0.012)$, with higher summary estimates for aggressive/lethal prostate cancer when restricted to Nordic studies that used medical record birthweight $\left(\mathrm{OR}_{\text {per } \mathrm{kg} \text { increase }}=1.37\right.$, $95 \% \mathrm{CI}=1.09,1.74)$, or studies in cohort/nested case-control designs $\left(\mathrm{OR}_{\text {per } \mathrm{kg} \text { increase }}=1.14,95 \% \mathrm{CI}=1.05,1.24\right)$ (Table 2). For comparison and comprehensiveness, we performed fixed-effects meta-analysis, in which we found a slightly smaller but statistically significant overall summary result $\left(\mathrm{OR}_{\text {per kg increase }}=1.04,95 \% \mathrm{CI}=1.00,1.08 ; P=0.026\right)$ as well as consistent subgroup results (Supplementary Table 4).

\section{DISCUSSION}

We did not find a statistically significant association between birthweight and total prostate cancer risk in the NSHD birth cohort, which may be because of the small number of events. Also, we did not have information on tumour characteristics to evaluate the association with aggressive/lethal prostate cancer in this birth cohort. However, our systematic review and meta-analysis suggests that greater birthweight is associated with small increased risks of total and aggressive/lethal prostate cancer. We detected a small amount of heterogeneity for total prostate cancer $\left(I^{2}=13 \%\right)$ but a moderate amount for aggressive/lethal disease $\left(I^{2}=40 \%\right)$. The associations of birthweight with total and aggressive/lethal prostate 


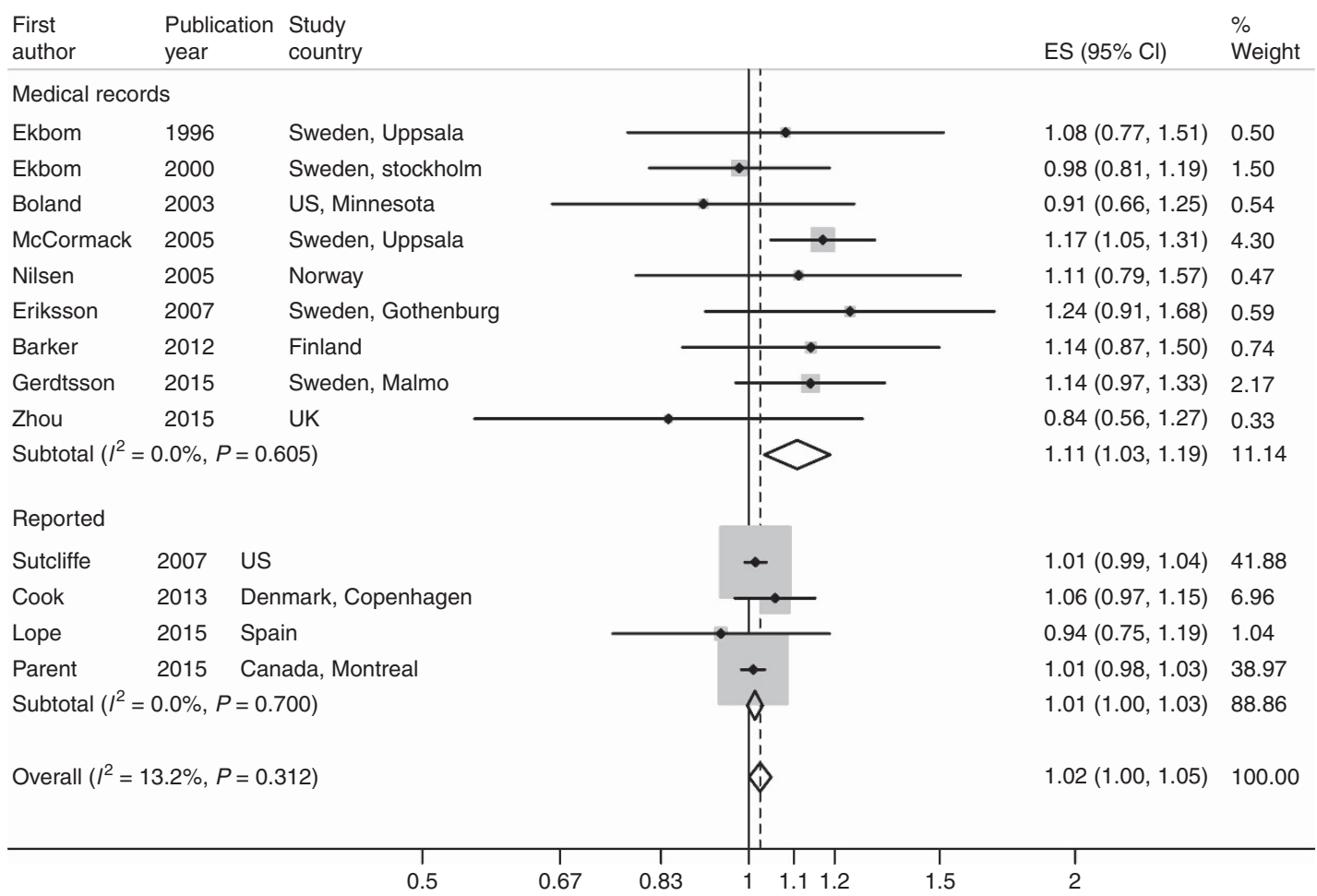

Figure 2. Birthweight (per $\mathrm{kg}$ ) in relation to total prostate cancer risk by birthweight source using a random-effects model $(N=13)$.

cancer were stronger when restricted to studies that used birthweight extracted from medical records or studies that were based in Nordic countries.

Although our meta-analysis estimated positive associations between birthweight and risks of total and aggressive/lethal prostate cancer, the magnitude of these associations was modest. However, we believe this modest magnitude is consistent with use of a proxy measure of the intrauterine environment rather than the possible causal exposure. Similar modest associations have also been observed for other in utero markers and prostate cancer risk, consistent with our findings for birthweight. For example, shorter duration of gestation, which is associated with lower birthweight, has been inversely associated with prostate cancer risk (Ekbom et al, 1996), whereas higher parity $(\geqslant 4)$ and placental weight, which are both associated with greater birthweight, have been positively associated with prostate cancer risk (Ekbom et al, 1996, 2000). In addition, taller adult height, another marker correlated with greater birthweight (Eide et al, 2005), was positively associated with total $\left(\mathrm{OR}_{\text {per } 5 \mathrm{~cm}}=1.04,95 \% \mathrm{CI}=1.03,1.05\right)$ and advanced $\left(\mathrm{OR}_{\text {per } 5 \mathrm{~cm}}=1.04,95 \% \mathrm{CI}=1.02,1.06\right)$ prostate cancer in a recent meta-analysis (World Cancer Research Fund International, 2014). Whether the link between birthweight and prostate cancer risks is mediated in full or part by adult height requires further investigation. Although birthweight is frequently used as a proxy of intrauterine exposures, the biologic mechanisms underlying a possible birthweight-prostate cancer association are not fully understood. Endogenous hormones, such as sex steroid hormones (Petridou et al, 1990; Peck et al, 2003) and IGFs (Orbak et al, 2001; Skalkidou et al, 2002; Davidson et al, 2006), and increased fetal stem cell burden (Strohsnitter et al, 2008; Capittini et al, 2011) in utero - all of which have demonstrated correlations with birthweight - have been hypothesised to modulate hormonal sensitivity and the risk of prostate cancer in adulthood.

Maternal and fetal sex steroid hormones act in concert in prenatal development, and homeostasis of both is essential for subsequent prostate health. The development of the prostate gland is androgen-dependent. Prostatic buds emerge from the urogenital sinus, which expresses androgen receptors stimulated by testicular androgens at approximately the tenth week of gestation (Cunha et al, 1987). It has been speculated that increased testosterone exposure in utero might reset the hypothalamic-pituitary-testicular feedback axis, leading to increased androgen secretion later in life. This hypothesis has been proposed to explain racial variation in prostate cancer risk (Ross and Henderson, 1994), as higher testosterone and oestradiol levels have been found in early gestational blood from African American than Caucasian women (Henderson et al, 1988; Potischman et al, 2005), higher testosterone to sex hormone-binding globulin concentration ratios have been found in cord blood from African American than their Caucasian male neonates (Rohrmann et al, 2009), and higher ageadjusted free testosterone concentrations were found in healthy adult African American men than their Caucasian counterparts in a recent meta-analysis (Richard et al, 2014). On the other hand, as fetal androgen levels decline and maternal oestrogen levels rise in the third trimester, exposure to excessive oestrogens can induce aberrant proliferation of the prostatic epithelium, which may lead to squamous metaplasia in the fetus, although this pathological change regresses rapidly after birth when maternal oestrogens plummet (Ellem and Risbridger, 2009). Evidence for oestrogen carcinogenesis has been consistently documented in rodent studies, which have demonstrated that exposure to high-dose diethylstilbestrol and $17 \beta$-oestradiol during development can reprogramme the prostate gland and increase its susceptibility to carcinogenesis with ageing (Prins and Ho, 2010).

Other hormones regulating growth and metabolism may also have roles in subsequent prostate carcinogenesis. Insulin-like growth factors are required for prostate gland development (Ruan et al, 1999), and adult circulating concentrations have been positively associated with prostate cancer risk (Rowlands et al, 2009). Moreover, IGF-1 and IGFBP-3 concentrations in cord blood have been correlated with increased number of stem cell measures, which may confer higher susceptibility to later malignant transformation, and to a lesser extent, oestradiol, oestriol and testosterone were also correlated with such (Baik et al, 2005). 
Table 2. Subgroup Analyses for birthweight (per $\mathrm{kg}$ ) in relation to prostate cancer risks by random-effects models

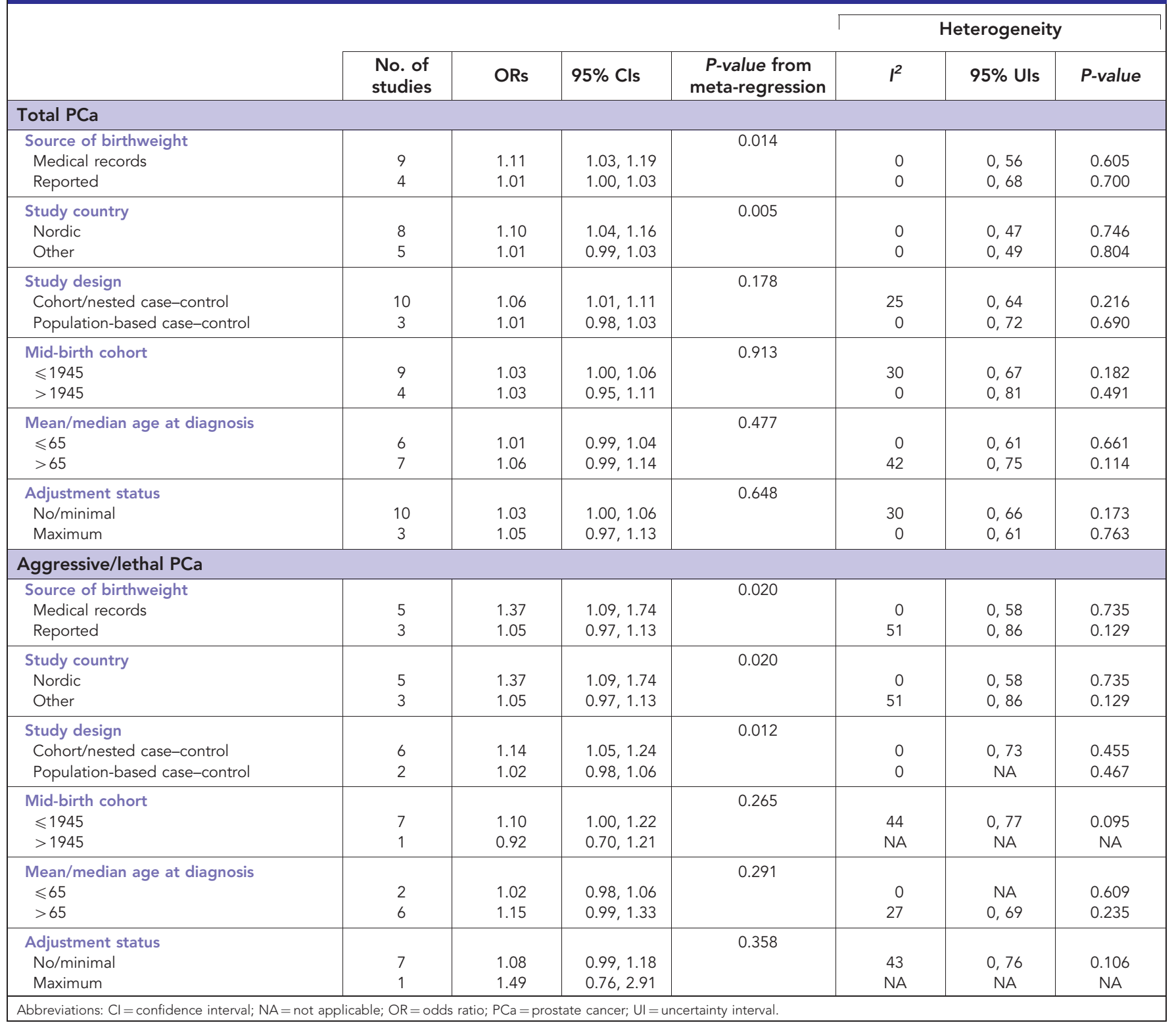

However, contrary to the expected direction of the association based on racial variation in prostate cancer risk, one study found that African-American neonates had lower concentrations of IGFs in cord blood than Caucasians (Rohrmann et al, 2009). Finally, leptin (Lai et al, 2011) and vitamin D (Eichholzer et al, 2013) cord blood levels were also similar in African-American and Caucasian neonates, suggesting that intrauterine exposure to these molecules is unlikely to explain racial differences in prostate cancer risk in adulthood.

This meta-analysis has several limitations that merit discussion. We cannot rule out measurement error in the three studies that used self-reported birthweight (Parent et al, 2008; Lope et al, 2012; Sutcliffe, 2007), given the fair-to-moderate agreement between selfreported and birth certificate-ascertained birthweight in prior validation studies (Jaworowicz et al, 2010); one cohort study that used maternal-reported birthweight (Cook et al, 2013) may have been subject to a lesser degree of measurement error (Adegboye and Heitmann, 2008). The stronger association observed in the subgroup of medical record-ascertained birthweight indirectly supports the possible existence of measurement error. Furthermore, we cannot exclude recall bias for two of these studies that ascertained birthweight information by self-report after prostate cancer diagnosis in a population-based case-control design (Parent et al, 2008; Lope et al, 2012), given that the summary estimate significantly increased by $10 \%\left(\mathrm{OR}_{\mathrm{per}} \mathrm{kg}\right.$ increase $=1.14,95 \%$ $\mathrm{CI}=1.05,1.24)$ for aggressive/lethal prostate cancer after omitting these two studies, although the summary estimate was not materially changed for total prostate cancer $\left(\mathrm{OR}_{\text {per } \mathrm{kg} \text { increase }}=1.05\right.$, $95 \% \mathrm{CI}=1.01,1.10 ; P=0.024)$. Measurement error and recall bias usually attenuate associations, thus we may have underestimated the magnitude of the relationships between birthweight and prostate cancer risks. Second, we extracted/estimated unadjusted or minimally adjusted risk estimates from the majority of studies included, even though gestational age, maternal smoking, pregnancy-related and neonatal complications (e.g., pre-eclampsia/ eclampsia and jaundice), birth order/parity and maternal anthropometric measures have been hypothesised to confound birthweight-prostate cancer associations. However, as prior studies to assess such factors found little or no effect on estimates (Boland et al, 2003; Nilsen et al, 2005; Eriksson et al, 2007; Zhou et al, herein), and as adjustment status was not significant in our subgroup analysis, we do not believe that use of minimally adjusted 


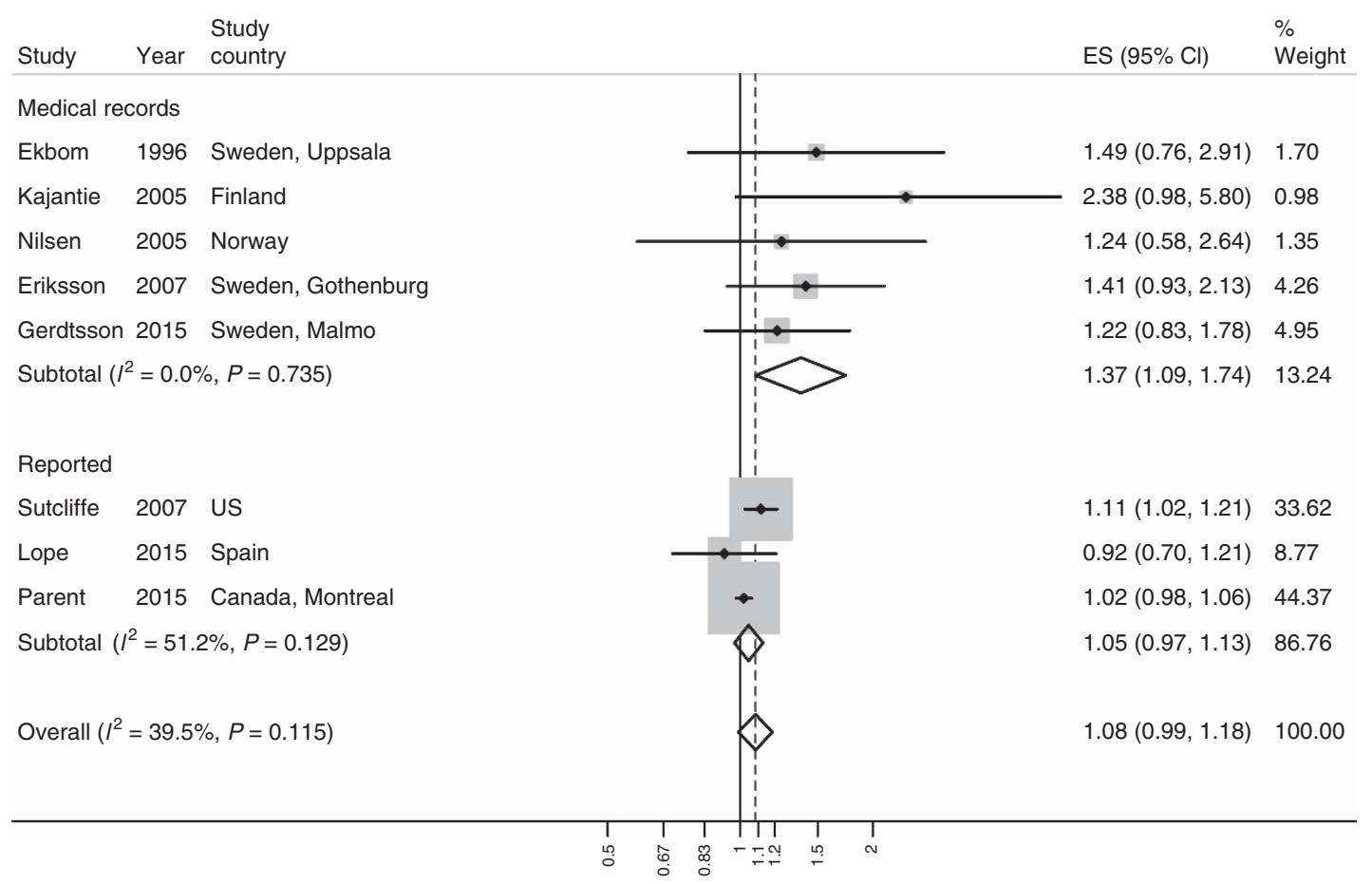

Figure 3. Birthweight (per $\mathrm{kg}$ ) in relation to aggressive/lethal prostate cancer risk by birthweight source using a random-effects model $(N=8)$.

estimates influenced our conclusions (Table 1). Third, longitudinal anthropometric measures, such as adult height, were only ascertained in two of the included studies (Cook et al, 2013; Gerdtsson et al, 2015). As these studies observed different results for anthropometric measures, we are unable to evaluate to what extent the effects of birthweight may be mediated by adult height. Fourth, we cannot exclude the possibility of publication bias for the meta-analysis on aggressive/lethal prostate cancer, given that only eight studies presented results for this subgroup of cases. Last, studies included in this meta-analysis were primarily conducted in men with European ancestry, and therefore we were unable to evaluate whether birthweight is associated with prostate cancer risks in men with African ancestry.

In conclusion, this systematic review and meta-analysis suggests that heavier birthweight may be associated with modest increased risks of total and aggressive/lethal prostate cancer. Novel approaches and longitudinal data are needed in future birth cohorts to elucidate biological mechanisms and determine the aetiological time windows for prostate carcinogenesis.

\section{ACKNOWLEDGEMENTS}

We thank Drs Johan G Erikson from the University of Helsinki, Finland; Virginia Lope from Carlos III Institute of Health, Spain; Clive Osmond from the University of Southampton, UK; MarieElise Parent from INRS-Institut Armand-Frappier, Canada; David Ulmert from Memorial Sloan-Kettering Cancer Center, USA as well as their colleagues for the additional information they generously provided about their publications on birth weight in relation to prostate cancer risks. We also thank Dr Barry I Graubard for his statistical consultation and support. This work was supported by the Intramural Program of the National Cancer Institute at the National Institutes of Health and Department of Health and Human Services. The findings and conclusions in this report are those of the authors and do not necessarily represent the official position of the National Institutes of Health.

\section{CONFLICT OF INTEREST}

The authors declare no conflict of interest.

\section{REFERENCES}

Adegboye ARA, Heitmann BL (2008) Accuracy and correlates of maternal recall of birthweight and gestational age. BJOG 115(7): 886-893.

Ahlgren M, Wohlfahrt J, Olsen LW, Sorensen TI, Melbye M (2007) Birth weight and risk of cancer. Cancer 110(2): 412-419.

Al Olama AA, Kote-Jarai Z, Berndt SI, Conti DV, Schumacher F, Han Y, Benlloch S, Hazelett DJ, Wang Z, Saunders E, Leongamornlert D, Lindstrom S, Jugurnauth-Little S, Dadaev T, Tymrakiewicz M, Stram DO, Rand K, Wan P, Stram A, Sheng X, Pooler LC, Park K, Xia L, Tyrer J, Kolonel LN, Le Marchand L, Hoover RN, Machiela MJ, Yeager M, Burdette L, Chung CC, Hutchinson A, Yu K, Goh C, Ahmed M, Govindasami K, Guy M, Tammela TLJ, Auvinen A, Wahlfors T, Schleutker J, Visakorpi T, Leinonen KA, Xu J, Aly M, Donovan J, Travis RC, Key TJ, Siddiq A, Canzian F, Khaw K-T, Takahashi A, Kubo M, Pharoah P, Pashayan N, Weischer M, Nordestgaard BG, Nielsen SF, Klarskov P, Røder MA, Iversen P, Thibodeau SN, McDonnell SK, Schaid DJ, Stanford JL, Kolb S, Holt S, Knudsen B, Coll AH, Gapstur SM, Diver WR, Stevens VL, Maier C, Luedeke M, Herkommer K, Rinckleb AE, Strom SS, Pettaway C, Yeboah ED, Tettey Y, Biritwum RB, Adjei AA, Tay E, Truelove A, Niwa S, Chokkalingam AP, Cannon-Albright L, Cybulski C, Wokołorczyk D, Kluźniak W, Park J, Sellers T, Lin H-Y, Isaacs WB, Partin AW, Brenner H, Dieffenbach AK, Stegmaier C, Chen C, Giovannucci EL, Ma J, Stampfer M, Penney KL, Mucci L, John EM, Ingles SA, Kittles RA, Murphy AB, Pandha H, Michael A, Kierzek AM, Blot W, Signorello LB, Zheng W, Albanes D, Virtamo J, Weinstein S, Nemesure B, Carpten J, Leske C, Wu S-Y, Hennis A, Kibel AS, Rybicki BA, Neslund-Dudas C, Hsing AW, Chu L, Goodman PJ, Klein EA, Zheng SL, Batra J, Clements J, Spurdle A, Teixeira MR, Paulo P, Maia S, Slavov C, Kaneva R, Mitev V, Witte JS, Casey G, Gillanders EM, Seminara D, Riboli E, Hamdy FC, Coetzee GA, Li Q, Freedman ML, Hunter DJ, Muir K, Gronberg H, Neal DE, Southey M, Giles GG, Severi G. The B, Prostate Cancer Cohort C, The PC, The CC, The G-ONECCook MB, Nakagawa H, Wiklund F, Kraft P, Chanock SJ, Henderson BE, Easton DF, Eeles RA, Haiman CA (2014) 
A meta-analysis of 87,040 individuals identifies 23 new susceptibility loci for prostate cancer. Nat Genet 46(10): 1103-1109.

Baik I, DeVito WJ, Ballen K, Becker PS, Okulicz W, Liu Q, Delpapa E, Lagiou P, Sturgeon S, Trichopoulos D, Quesenberry PJ, Hsieh CC (2005) Association of fetal hormone levels with stem cell potential: evidence for early life roots of human cancer. Cancer Res 65(1): 358-363.

Barker DJ, Osmond C, Thornburg KL, Kajantie E, Eriksson JG (2012) A possible link between the pubertal growth of girls and prostate cancer in their sons. Am J Hum Biol 24(4): 406-410.

Boland LL, Mink PJ, Bushhouse SA, Folsom AR (2003) Weight and length at birth and risk of early-onset prostate cancer (United States). Cancer Causes Control 14(4): 335-338.

Capittini C, Bergamaschi P, De Silvestri A, Marchesi A, Genovese V, Romano B, Tinelli C, Salvaneschi L (2011) Birth-weight as a risk factor for cancer in adulthood: the stem cell perspective. Maturitas 69(1): 91-93.

Chene G, Thompson SG (1996) Methods for summarizing the risk associations of quantitative variables in epidemiologic studies in a consistent form. Am J Epidemiol 144(6): 610-621.

Cook MB, Gamborg M, Aarestrup J, Sorensen TI, Baker JL (2013) Childhood height and birth weight in relation to future prostate cancer risk: a cohort study based on the copenhagen school health records register. Cancer Epidemiol Biomarkers Prev 22(12): 2232-2240.

Cunha GR, Donjacour AA, Cooke PS, Mee H, Bigsby RM, Higgins SJ, Sugimura Y (1987) The endocrinology and developmental biology of the prostate. Endocr Rev 8(3): 338-362.

Davidson S, Hod M, Merlob P, Shtaif B (2006) Leptin, insulin, insulin-like growth factors and their binding proteins in cord serum: insight into fetal growth and discordancy. Clin Endocrinol 65(5): 586-592.

dos Santos Silva I, Stavola BD, McCormack V. Collaborative Group on PreNatal Risk F, Subsequent Risk of Breast C (2008) Birth size and breast cancer risk: re-analysis of individual participant data from 32 studies. PLoS Med 5(9): e193.

Eichholzer M, Platz EA, Bienstock JL, Monsegue D, Akereyeni F, Hollis BW, Horst R, Rifai N, Pollak MN, Barbir A, Agurs-Collins T, Rohrmann S (2013) Racial variation in vitamin D cord blood concentration in white and black male neonates. Cancer Causes Control 24(1): 91-98.

Eide MG, Oyen N, Skjaerven R, Nilsen ST, Bjerkedal T, Tell GS (2005) Size at birth and gestational age as predictors of adult height and weight. Epidemiology 16(2): 175-181.

Ekbom A (1998) Growing evidence that several human cancers may originate in utero. Semin Cancer Biol 8(4): 237-244.

Ekbom A, Hsieh CC, Lipworth L, Wolk A, Ponten J, Adami HO, Trichopoulos D (1996) Perinatal characteristics in relation to incidence of and mortality from prostate cancer. BMJ 313(7053): 337-341.

Ekbom A, Wuu J, Adami HO, Lu CM, Lagiou P, Trichopoulos D, Hsieh C (2000) Duration of gestation and prostate cancer risk in offspring. Cancer Epidemiol Biomarkers Prev 9(2): 221-223.

Ellem SJ, Risbridger GP (2009) The dual, opposing roles of estrogen in the prostate. Ann NY Acad Sci 1155: 174-186.

Eriksson M, Wedel H, Wallander MA, Krakau I, Hugosson J, Carlsson S, Svardsudd K (2007) The impact of birth weight on prostate cancer incidence and mortality in a population-based study of men born in 1913 and followed up from 50 to 85 years of age. Prostate 67(11): 1247-1254

Gerdtsson A, Poon JB, Thorek DL, Mucci LA, Evans MJ, Scardino P, Abrahamsson PA, Nilsson P, Manjer J, Bjartell A, Malm J, Vickers A, Freedland SJ, Lilja H, Ulmert D (2015) Anthropometric measures at multiple times throughout life and prostate cancer diagnosis, metastasis, and death. Eur Urol 17(15): 00226-2.

Henderson BE, Bernstein L, Ross RK, Depue RH, Judd HL (1988) The early in utero oestrogen and testosterone environment of blacks and whites: potential effects on male offspring. Br J Cancer 57(2): 216-218.

Jaworowicz DJJ, Nie J, Bonner MR, Han D, Vito D, Hutson A, Potischman N, Trevisan M, Muti P, Freudenheim JL (2010) Agreement between self-reported birth weight and birth certificate weights. J Devl Orig Health Dis 1(02): 106-113.

Kajantie E, Osmond C, Barker DJ, Forsen T, Phillips DI, Eriksson JG (2005) Size at birth as a predictor of mortality in adulthood: a follow-up of 350 000 person-years. Int J Epidemiol 34(3): 655-663.

Kuh D, Ben-Shlomo Y (2004) A Life Course Approach to Chronic Disease Epidemiology: Tracing the Origins of Ill-Health from Early to Adult Life. 2nd edn, Fully revised. Oxford University Press: Oxford, UK.
Lahmann PH, Lissner L, Wallstrom P, Gullberg B, Olsson H (2010) Birth weight and current body size in relation to risk of prostate cancer: the Malmo Diet and Cancer Study, Sweden. Obes Rev 11: 354-355.

Lahmann PH, Wallstrom P, Lissner L, Olsson H, Gullberg B (2012) Measures of birth size in relation to risk of prostate cancer: the Malmo Diet and Cancer Study, Sweden. J Dev Orig Health Dis 3(6): 442-449.

Lai GY, Rohrmann S, Agurs-Collins T, Sutcliffe CG, Bradwin G, Rifai N, Bienstock JL, Platz EA (2011) Racial variation in umbilical cord blood leptin concentration in male babies. Cancer Epidemiol Biomarkers Prev 20(4): 665-671.

Lawson JS (1998) Prostate cancer, birthweight, and diet. Epidemiology 9(2): 217.

Lope V, Garcia-Esquinas E, Aragones N, Kogevinas M, Dierssen-Sotos T, Altzibar J, Ardanaz E, Martin V, Tardon A, Alguacil J, Crous-Bou M, Salas D, Jimenez-Moleon JJ, Pollan M (2012) Birth and childhood characteristics and risk of adult hormone dependent cancers:MCC-Spain. Epidemiology 23(5): S510.

McCormack VA, dos Santos Silva I, Koupil I, Leon DA, Lithell HO (2005) Birth characteristics and adult cancer incidence: Swedish cohort of over 11,000 men and women. Int J Cancer 115(4): 611-617.

Nilsen TI, Romundstad PR, Troisi R, Vatten LJ (2005) Birth size and subsequent risk for prostate cancer: a prospective population-based study in Norway. Int J Cancer 113(6): 1002-1004.

Orbak Z, Darcan S, Coker M, Goksen D (2001) Maternal and fetal serum insulin-like growth factor-I (IGF-I) IGF binding protein-3 (IGFBP-3), leptin levels and early postnatal growth in infants born asymmetrically small for gestational age. J Pediatr Endocrinol Metab 14(8): 1119-1127.

Parent ME, Siemiatycki J, Goldberg M, Desy M (2008) Birthweight, obesity during childhood, adolescence and adulthood, and prostate cancer preliminary data from the PROTEuS study. Am J Epidemiol 167(11): S62-S62.

Peck JD, Hulka BS, Savitz DA, Baird D, Poole C, Richardson BE (2003) Accuracy of fetal growth indicators as surrogate measures of steroid hormone levels during pregnancy. Am J Epidemiol 157(3): 258-266.

Petridou E, Panagiotopoulou K, Katsouyanni K, Spanos E, Trichopoulos D (1990) Tobacco smoking, pregnancy estrogens, and birth weight. Epidemiology 1(3): 247-250.

Platz EA, Giovannucci E, Rimm EB, Curhan GC, Spiegelman D, Colditz GA, Willett WC (1998) Retrospective analysis of birth weight and prostate cancer in the Health Professionals Follow-up Study. Am J Epidemiol 147(12): 1140-1144.

Potischman N, Troisi R, Thadhani R, Hoover RN, Dodd K, Davis WW, Sluss PM, Hsieh CC, Ballard-Barbash R (2005) Pregnancy hormone concentrations across ethnic groups: implications for later cancer risk. Cancer Epidemiol Biomarkers Prev 14(6): 1514-1520.

Prins GS, Ho SM (2010) Early-life estrogens and prostate cancer in an animal model. J Dev Orig Health Dis 1(6): 365-370.

Richard A, Rohrmann S, Zhang L, Eichholzer M, Basaria S, Selvin E, Dobs AS, Kanarek N, Menke A, Nelson WG, Platz EA (2014) Racial variation in sex steroid hormone concentration in black and white men: a meta-analysis. Andrology 2(3): 428-435.

Rohrmann S, Sutcliffe CG, Bienstock JL, Monsegue D, Akereyeni F, Bradwin G, Rifai N, Pollak MN, Agurs-Collins T, Platz EA (2009) Racial variation in sex steroid hormones and the insulin-like growth factor axis in umbilical cord blood of male neonates. Cancer Epidemiol Biomarkers Prev 18(5): 1484-1491.

Ross RK, Henderson BE (1994) Do diet and androgens alter prostate cancer risk via a common etiologic pathway? J Natl Cancer Inst 86(4): $252-255$.

Rowlands M-A, Gunnell D, Harris R, Vatten LJ, Holly JMP, Martin RM (2009) Circulating insulin-like growth factor (IGF) peptides and prostate cancer risk: a systematic review and meta-analysis. Int J Cancer 124(10): 2416-2429.

Ruan W, Powell-Braxton L, Kopchick JJ, Kleinberg DL (1999) Evidence that insulin-like growth factor I and growth hormone are required for prostate gland development. Endocrinology 140(5): 1984-1989.

Shibata A, Minn AY (2000) Perinatal sex hormones and risk of breast and prostate cancers in adulthood. Epidemiol Rev 22(2): 239-248.

Skalkidou A, Petridou E, Papathom E, Salvanos H, Chrousos G, Trichopoulos D (2002) Birth size and neonatal levels of major components of the IGF system: implications for later risk of cancer. J Pediatr Endocrinol Metab 15(9): 1479-1486. 
Strohsnitter WC, Savarese TM, Low HP, Chelmow DP, Lagiou P, Lambe M, Edmiston K, Liu Q, Baik I, Noller KL, Adami HO, Trichopoulos D, Hsieh CC (2008) Correlation of umbilical cord blood haematopoietic stem and progenitor cell levels with birth weight: implications for a prenatal influence on cancer risk. Br J Cancer 98(3): 660-663.

Sutcliffe S, Colditz GA (2013) Prostate cancer: is it time to expand the research focus to early-life exposures? Nat Rev Cancer 13(3): 208-518.

Sutcliffe S, Giovannucci E, Willett WC, Platz EA (2007) A prospective study of birth weight and risk of prostate cancer. Proc Am Assoc Cancer Res Annu Meet 48: 820-820.

Tibblin G, Eriksson M, Cnattingius S, Ekbom A (1995) High birthweight as a predictor of prostate cancer risk. Epidemiology 6(4): 423-424.
Wadsworth M, Kuh D, Richards M, Hardy R (2006) Cohort Profile: The 1946 National Birth Cohort (MRC National Survey of Health and Development). Int J Epidemiol 35(1): 49-54.

World Cancer Research Fund International (2014) Continuous Update Project Report: Diet, Nutrition, Physical Activity, and Prostate Cancer. Available at: www.wcrf.org/sites/default/files/Prostate-Cancer-2014-Report.pdf (accessed on 8 July 2015).

This work is published under the standard license to publish agreement. After 12 months the work will become freely available and the license terms will switch to a Creative Commons AttributionNonCommercial-Share Alike 4.0 Unported License.

Supplementary Information accompanies this paper on British Journal of Cancer website (http://www.nature.com/bjc) 